\title{
Real-Time Simulation of Medical Ultrasound from CT Images
}

\author{
Ramtin Shams ${ }^{1}$, Richard Hartley ${ }^{1,2}$, and Nassir Navab ${ }^{3}$ \\ ${ }^{1}$ RSISE, The Australian National University, Canberra \\ ${ }^{2}$ NICTA, Canberra, Australia ${ }^{\star}$ \\ ${ }^{3}$ Computer Aided Medical Procedures (CAMP), TU München, Germany
}

\begin{abstract}
Medical ultrasound interpretation requires a great deal of experience. Real-time simulation of medical ultrasound provides a costeffective tool for training and easy access to a variety of cases and exercises. However, fully synthetic and realistic simulation of ultrasound is complex and extremely time-consuming. In this paper, we present a novel method for simulation of ultrasound images from 3D CT scans by breaking down the computations into a preprocessing and a run-time phase. The preprocessing phase produces detailed fixed-view 3D scattering images and the run-time phase generates view-dependent ultrasonic artifacts for a given aperture geometry and position within a volume of interest. We develop a simple acoustic model of the ultrasound for the run-time phase, which produces realistic ultrasound images in real-time when combined with the previously computed scattering image.
\end{abstract}

\section{Introduction}

Ultrasound is a challenging imaging modality to master due to a multitude of parameters involved in acquisition and interpretation of resulting images. Real-time simulation of ultrasound can be used for training to complement a theoretical study program. A recent study in 1] reports a significant improvement in skills of subjects who received additional simulator-based training. The main advantage is easy access to a wealth of standard and rare cases collected over time.

Existing methods for real-time simulation of ultrasound such as [2]3/4 are based on acquisition of actual ultrasound images and creating a compounded 3D volume of the region of interest. The volume is then sliced at run-time based on the position of a virtual probe. The resulting images are realistic as long as the operator navigates close to the acquisition positions. As the probe is navigated further away from the acquisition positions, the images become less realistic, since view-dependent ultrasound effects are no longer accurately represented. The acquisition process is also complicated. One needs to ensure that the volume of interest is imaged from various positions and does not contain view-dependent artifacts such as shadowing, and the effect of a fixed gain and focus.

* NICTA is a research centre funded by the Australian Government through Backing Australia's Ability and the ICT Research Centre of Excellence programs. 
In this paper, we use the term ultrasound simulation in a slightly different context, referring to the process of creating fully synthetic ultrasound images. We synthesize the ultrasound image from a fixed-view scattering image and a view-dependent reflection image. The scattering image is generated off-line using Field II [5,6] and the reflection image is based on a simple model for acoustic wave propagation in a piecewise homogenous medium and is generated in realtime. Both images are derived from a 3D CT scan of the region of interest.

An ultrasound simulator based on CT scans was claimed earlier by [7. We are unable to assess the method's performance, since the paper does not divulge much information, citing a pending patent. However, the only result shown in the paper, illustrates that many ultrasound effects are not simulated and that further work is of value.

A simple ultrasound model that can be implemented in real-time is used in 8 for CT to ultrasound registration. The method produces convincing registration results without the need for highly realistic ultrasound simulations. This is not an issue for the intended application since a novel multi-modal registration method is also introduced to compensate for the limitations of the simulated ultrasound.

\section{Method}

\subsection{A Simple Acoustic Model for Ultrasound}

We develop a simple acoustic model that can be used in real-time for simulation of large-scale reflections, attenuation due to reflections, effect of a finite beamwidth, and view-dependent shadow and occlusion effects in an ultrasound image.

Reflection. A sound beam traveling through a piecewise homogenous medium is partially reflected at the interface of two media with differing acoustic impedances. This impedance mismatch is the primary mechanism that allows visualization with ultrasound. The acoustic impedance is defined by $Z=\rho c$, where $\rho$ is the density of the medium and $c$ is the speed of sound. The ratio of reflected energy to incident energy is called the reflection coefficient, $\alpha_{R}$, and is given by

$$
\alpha_{R}=\left(\frac{Z_{2}-Z_{1}}{Z_{2}+Z_{1}}\right)^{2},
$$

at the interface of the two media with acoustic impedances $Z_{1}$ and $Z_{2}$ [9].

Lambertian Scattering. Reflection of sound beams at an interface is the main interaction of interest to us. The reflection is typically non-specular and subject to scattering. The intensity of the scattered signal (from a receiver's point of view) depends on the angle of incidence and is maximal for a beam normal to the interface and approaches zero as the incident angle approaches $90^{\circ}$. This effect can be described by Lambertian scattering 1 . The intensity of the reflected

\footnotetext{
${ }^{1}$ We use a Lambertian model for its simplicity. A more appropriate model for ultrasound scattering is Rayleigh scattering, since the interface dimensions are much smaller than a wavelength 9. This is the subject of further investigation.
} 
signal at a point $\mathbf{x}$ on the interface of the media depends on the angle of incidence and can be written as

$$
R(\mathbf{x})=\alpha_{R}(\mathbf{x}) I_{i}(\mathbf{x})|\mathbf{r}(\mathbf{x}) \cdot \mathbf{n}(\mathbf{x})|,
$$

where $I_{i}(\cdot)$ is the intensity of the incident beam at the interface, $\mathbf{r}$ is the unit vector in the direction of the beam, $\mathbf{n}$ is the surface normal, $|\cdot|$ is the absolute value operator, and $R(\cdot)$ is the intensity of the reflected signal. According to the Lambertian scattering model, the intensity of the reflected signal, perceived by an arbitrary viewer, is independent of the view angle and only depends on the angle of incidence. This is of course provided that no attenuation occurs in the return path between the point of reflection and the observer. If the initial intensity of the transmitted signal is shown by $I_{0}$, the attenuation coefficient at point $\mathbf{x}$ is given by $I_{i}(\mathbf{x}) / I_{0}$. Since the reflected signal travels back through the same attenuating medium (ignoring any refraction), the intensity of the signal as sensed by the receiver, $I_{r}(\mathbf{x})$, is attenuated by the same coefficient as in the forward path and can be written as

$$
I_{r}(\mathbf{x}) \propto \alpha_{R}(\mathbf{x}) \frac{I_{i}^{2}(\mathbf{x})}{I_{0}}|\mathbf{r}(\mathbf{x}) \cdot \mathbf{n}(\mathbf{x})| .
$$

Effect of Beam Width. The aperture of an ultrasound transducer consists of a number of acoustic elements. Typically a group of adjacent elements are actively sending and receiving acoustic signals while others are turned off (see Fig.1 a). Using a group of active elements produces a deeper near field and a less diverging far field compared to a single element acting alone 9]. The active aperture is electronically shifted along the aperture to cover the entire field of view. This results in the transmitted signal from a single element to be partially received by adjacent elements. One novel aspect of the present paper is modeling this effect. The reflected signal is integrated along the active wavefront at a specified depth using a suitable window function which results in a more realistic reflection image (see Fig.2). For a linear array transducer we can write

$$
I_{r}(x, y) \propto \int_{x-\ell}^{x+\ell} \alpha_{R}(u, y) \frac{I_{i}^{2}(u, y)}{I_{0}}|\mathbf{r}(u, y) \cdot \mathbf{n}(u, y)| \omega(u) d u,
$$

where $\omega(\cdot)$ is the window function, and $\ell$ is length of the active aperture, given by $\ell=n_{a}\left(w_{e}+s_{e}\right)$, where $n_{a}$ is the number of active elements, $w_{e}$ is the width of each element, and $s_{e}$ is the spacing between adjacent elements. If the active aperture is moved at a constant frequency, $f_{a}$, each element will be active for a period of time equal to $\left(2 n_{a}-1\right) / f_{a}$. Let us consider the $m^{\text {th }}$ element in the transducer: during its operating interval, it will receive reflections due to operation of elements in the range $m-n_{a}+1$ to $m+n_{a}-1$. The amount of reflected signal due to an element $i \in\left[m-n_{a}+1, m+n_{a}-1\right]$ is proportional to the amount of time when $i$ and $m$ are both turned on, which is given by

$$
t(i)=\frac{2 n_{a}-1}{f_{a}}\left(1-\left|\frac{i-m}{n_{a}}\right|\right) .
$$



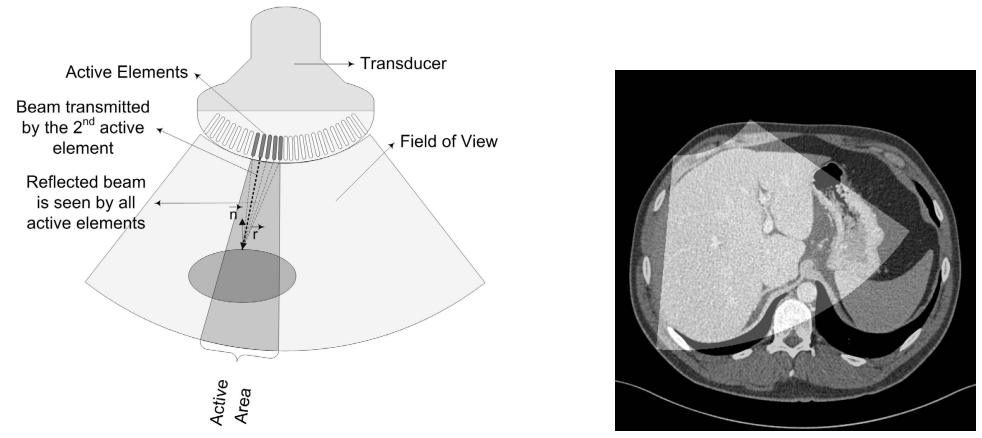

Fig. 1. From left: (a) A convex array transducer with a multi-element active aperture, (b) Ultrasound field of view superimposed on the liver of a human subject

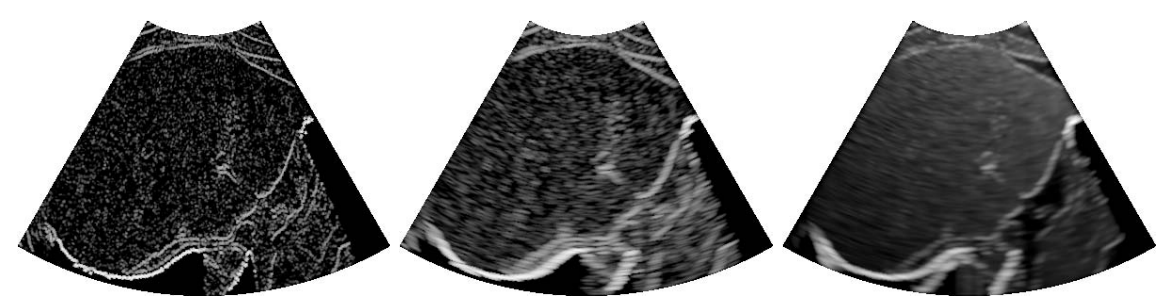

Fig. 2. From left: (a) Reflection image with one active element, (b) Reflection image with multiple active elements, (c) Our simulated ultrasound (combined reflection and scattering images.), Note: images depict the region of interest shown in Fig.11 and are log-compressed. Also notice shadowing on the right-hand side due to an air-tissue interface and in the middle-bottom of the image due to a a bone-tissue interface.

This results in a triangular window function $\omega(\cdot)$ in (4). In our simulations, though, we used a Hann window to further suppress the contributions from elements that are farthest away.

Note that thanks to modeling the beam width effect, one can infer the direction of ultrasound beams by looking at the content of Fig.2 $\mathrm{b}$ and Fig.22.

\subsection{Creating the Reflection Image}

The reflection image simulates view-dependent ultrasonic effects due to reflection and attenuation of the signal. Tissue boundaries are emphasized in the image and shadows due to large impedance mismatches between tissue-bone and tissue-air interfaces are simulated (refer to Fig.21).

We use a CT volume for real-time simulation of the reflection image. An edge volume, based on the method proposed in [10] with a Deriche filter, is computed from the CT image. Edge detection needs to be performed once, when the CT image is first loaded. Given a set of acquisition parameters and position information, a corresponding plane from the CT and edge volumes is extracted. 

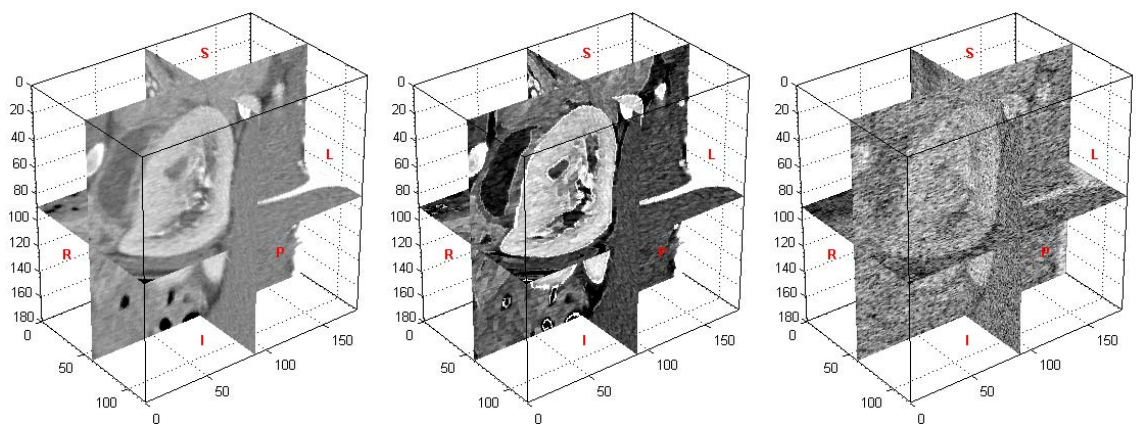

Fig. 3. From left: (a) CT scan of the kidney of an animal subject, (b) Contrast-adjusted and edge-enhanced CT image used as a scattering map, (c) The scattering image generated by Field II

The $3 \mathrm{D}$ edge volume removes the need for $2 \mathrm{D}$ edge detection at run-time and also provides better continuity as the probe is navigated.

The implementation of the algorithm for a linear transducer is straightforward. Transmission and reflection coefficients and angles of incidence are calculated at the interface between every two media along an axial scan line. The interfaces are detected from the edge map. The acoustic impedances of the media, divided by each interface, are determined from the average intensity of the CT image along the scan line between the interface boundaries. The CT intensities for tissues are approximately proportional to the acoustic impedance for tissues [8] and can be used directly for calculation of the reflection coefficients in (11). This is not true for bone and air, so we also label the media as bone, air and tissue based on their CT Hounsfield intensities. On average bone-tissue interfaces reflect $43 \%$ and air-tissue interfaces reflect $99 \%$ of the incident beam [9].

The process of simulating linear array and convex array transducers are similar with the exception that for convex array transducers, the extracted CT slice has a fan-shaped field of view. We first warp the fan-shaped area to a rectangle using a Cartesian to polar transformation. This, in turn, transforms the convex transducer to a linear one and allows us to use (4) for both geometries.

\subsection{Creating the Scattering Image}

Realistic speckle patterns can be simulated using software packages such as Field II [5]. Simulations are based on the principles of linear acoustics and computation of the spatial impulse response [11. Speckle is simulated by randomly placed scatterers with strength randomly chosen by Field II from a normal distribution. The mean of this distribution is location-dependent, and is provided as input to the simulator in the form of a scattering map, which gives the mean scatterer strength at all points within the volume of interest. A typical B-mode image requires anywhere from 200,000 to $1,000,000$ point scatterers in order to create a realistic speckle pattern. Scattering simulation in this way is computationally 
expensive. On a standard PC, with 1,000,000 point scatterers, simulation of a single ultrasound beam takes almost 20 minutes. This is nearly 2 days for a B-mode image with $128 \mathrm{RF}$ scan lines. For this reason the scattering image is preprocessed from a single view and stored along with the CT image.

Direct use of a CT image as a scattering map results in a repetitive scattering pattern where hardly any structures are recognizable. We overcome this by using a contrast- and edge-enhanced image (shown in Fig.3) as our scattering map. First the CT image undergoes affine contrast-stretching to maximize contrast while allowing no more than $5 \%$ of voxels to under- or overflow (saturate) the intensity range. The resulting scattering map is then further enhanced by emphasizing tissue or organ boundaries, which represent highly scattering areas. This is done using the previously calculated edge-map; detected edge points are set to maximum value in the scattering map. Fig. 3 shows 3 -view images of the original CT image, the edge- and contrast-enhanced scattering map, and the resulting scattering image which exhibits speckle.

The 3D scattering volume is simulated slice-by-slice in the axial plane. A virtual linear array transducer operating at $7.5 \mathrm{MHz}$, is positioned along the leftright (LR)-axis of the slice. The speed of sound is assumed to be $c=1540 \mathrm{~m} / \mathrm{s}$ resulting in a wavelength of $\lambda=205.3 \mu \mathrm{m}$. Width of each transducer element is set to $\lambda$. Kerf (spacing between elements) is set to $0.1 \lambda$. The aperture length is slightly longer than the image width. In our experiments, this results in an aperture with 504 elements. There are 64 active elements in the aperture. 128 RF scan lines were simulated per slice.

We used a 20 CPU cluster to parallelize the computations for a $180 \times 120 \times 180$ pixels volume of interest, depicting the kidney of an animal subject, cropped from a larger CT image with a spacing of $0.55 \times 0.55 \times 0.60 \mathrm{~mm}$. Four scatterers were introduced per voxel resulting in a total of $15,552,000$ scatterers for the entire volume and a total simulation time of nearly 32 hours.

\section{Creating the Ultrasound Image}

The final simulation is the result of combining scattering and reflection images:

$$
I_{u s}(\mathbf{x})=\left(I_{r}(\mathbf{x})+\alpha\right) I_{s}(\mathbf{x}),
$$

where $I_{u s}(\cdot)$ is the ultrasound image, $I_{r}(\cdot)$ is the reflection image and $I_{s}(\cdot)$ is the elongated scattering image. We elongate the scattering image orthogonal to the beam direction using a window function similar to equation (4). Increasing $\alpha$, results in a stronger speckle texture, while reducing it makes reflections more dominant. The operator selects a suitable level for $\alpha$, as well as, an appropriate level of log-compression (to limit the dynamic range of the signal) for best visual results.

Fig.4 shows an oblique plane within the CT volume and corresponding scattering and simulated ultrasound images. Note the highly reflective areas in the ultrasound around the kidney boundaries and vasculature and the realistic speckle pattern. The tumor can be easily located in the simulated ultrasound. 

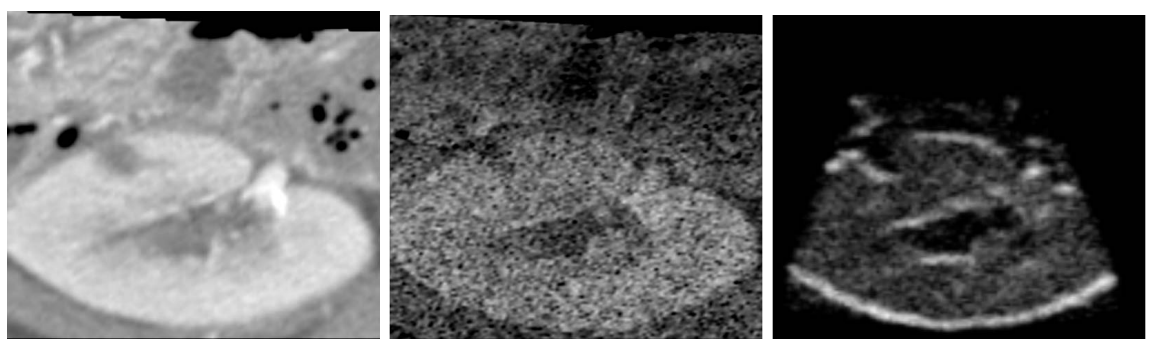

Fig. 4. From left: (a) CT scan of an oblique plane within the CT volume, (b) Scattering image generated by Field II, (c) Our simulated ultrasound image: note the speckle pattern and strong reflection from kidney boundaries. The tumor on the top-left corner of the kidney can be easily identified.

$\mathrm{A} \mathrm{C}++$ implementation of our algorithm (without any particular attention to optimization of the code e.g. use of SIMD instruction set) can generate images similar to Fig.2r at 10-15 frames/sec.

\section{Discussion}

We are investigating further enhancement of our ultrasound model including absorbtion and refraction based on labeling tissue types, multiple echoes, and simulating tissue deformation due to pressure by the ultrasound probe.

One limitation of the method is that it may not be readily used for prenatal training due to dependance on CT images as input. Prenatal CT scans are rarely performed and are typically reserved for cases with complications or when the fetus is deemed unviable. Use of MRI or synthetic models can be investigated.

Another interesting application of ultrasound simulation is real-time registration of $2 \mathrm{D}$ ultrasound to a $3 \mathrm{D}$ volume. We are hypothesizing that a more accurate simulation of the ultrasound, facilitates registration of an actual ultrasound against a CT volume. This is driven by the intuition that comparing an actual ultrasound with a closely simulated one, reduces the burden on the design of the similarity measure and the optimization algorithm and can potentially lead to interactive registration. This application is currently being investigated.

Simulating the scattering image is time-consuming and requires a cluster of CPUs to be practical. This is less of a burden as 4- and 8-core systems are becoming commonplace. GPU implementation of the scattering simulation is worthwhile as GPUs with up to 128-cores are available at retail prices and the processing can be easily distributed. From experience [12 13], GPU-based implementation of algorithms that are amenable to parallelization can receive a 10 to 30 fold improvement in execution times over a single-core CPU. 


\section{Acknowledgements}

Part of the data-set used in our experiments was kindly provided by Prof. Kirby Vosburgh. The authors wish to sincerely thank Prof. Vosburgh for the contribution. The first author is also indebted to Mr. James Ashton and Mr. Nic Boling for their exceptional effort in preparing the computational resources needed for the project, in a timely manner.

\section{References}

1. Maul, H., Scharf, A., Baier, P., Wüstemann, M., Günter, H.H., Gebauer, G., Sohn, C.: Ultrasound simulators: experience with the sonotrainer and comparative review of other training systems. Ultrasound Obstet. Gynecol. 24(5), 581-585 (2004)

2. Aiger, D., Cohen-Or, D.: Real-time ultrasound imaging simulation. Real-Time Imag. 4(4), 263-274 (1998)

3. Terkamp, C., Kirchner, G., Wedemeyer, J., Dettmer, A., Kielstein, J., Reindell, H., Bleck, J., Manns, M., Gebel, M.: Simulation of abdomen sonography. evaluation of a new ultrasound simulator. Ultraschall in Med. 24, 239-244 (2003)

4. Henry, D., Troccaz, J., Bosson, J.L., Pichot, O.: Ultrasound imaging simulation: Application to the diagnosis of deep venous thromboses of lower limbs. In: Wells, W.M., Colchester, A.C.F., Delp, S.L. (eds.) MICCAI 1998. LNCS, vol. 1496, pp. 1032-1040. Springer, Heidelberg (1998)

5. Jensen, J.A.: Field: A program for simulating ultrasound systems. In: 10th NordicBaltic Conference on Biomedical Imaging Published in Medical \& Biological Engineering \& Computing, pp. 351-353 (1996)

6. Jensen, J.A., Svendsen, N.B.: Calculation of pressure fields from arbitrarily shaped, apodized, and excited ultrasound transducers. IEEE Trans. Ultrason. 39, 262-267 (1992)

7. Hostettler, A., Forest, C., Forgione, A., Soler, L., Marescaux, J.: Real-time ultrasonography simulator based on 3D CT-scan images. Medicine Meets Virtual Reality (MMVR) 111 (February 2005)

8. Wein, W., Khamene, A., Clevert, D.A., Kutter, O., Navab, N.: Simulation and fully automatic multimodal registration of medical ultrasound. In: Ayache, N., Ourselin, S., Maeder, A. (eds.) MICCAI 2007, Part I. LNCS, vol. 4791, pp. 136-143. Springer, Heidelberg (2007)

9. Hedrick, W., Hykes, D., Starchman, D.: Ultrasound Physics and Instrumentation, 3rd edn. Mosby - Year Book, Inc. (1995)

10. Monga, O., Deriche, R., Malandain, G., Cocquerez, J.P.: Recursive filtering and edge tracking: two primary tools for $3 \mathrm{~d}$ edge detection. Image and Vision Computing 9(4), 203-214 (1991)

11. Stepanishen, P.R.: Transient radiation from pistons in an infinite planar baffle. J. Acoust. Soc. Am. 49, 1629-1638 (1971)

12. Shams, R., Barnes, N.: Speeding up mutual information computation using NVIDIA CUDA hardware. In: Proc. Digital Image Computing: Techniques and Applications (DICTA), Adelaide, Australia, December 2007, pp. 555-560 (2007)

13. Shams, R., Kennedy, R.A.: Efficient histogram algorithms for NVIDIA CUDA compatible devices. In: Proc. Int. Conf. on Signal Processing and Communications Systems (ICSPCS), Gold Coast, Australia, December 2007, pp. 418-422 (2007) 